\title{
Dedicated model for the efficient assessment of wireless power transfer in the radiative near-field
}

\author{
Gert-Jan Stockman*, ${ }^{*}$, Hendrik Rogier and Dries Vande Ginste \\ IBCN/Electromagnetics Group, Department of Information Technology, Ghent University/iMinds, \\ Sint-Pietersnieuwstraat 41, Ghent, 9000, Belgium
}

\begin{abstract}
SUMMARY
In this paper, a novel method to efficiently calculate the power transfer efficiency of a wireless power transfer system in the radiative near-field is proposed. The technique allows repositioning of the antennas without large additional cost. It relies on a single simulation (or measurement) of the radiation pattern of the antennas used. Thanks to its high computational efficiency, it can be used in multi-transmitter and multi-receiver scenarios when there is no coupling between devices via the reactive near-field. Our method is applied to the latter to demonstrate its accuracy and computational efficiency. Copyright (C) 2015 John Wiley \& Sons, Ltd.
\end{abstract}

Received 29 July 2014; Revised 11 March 2015; Accepted 22 April 2015

KEY WORDS: $\quad$ wireless power transfer; power transfer efficiency; radiative near-field; multi-receiver scenario

\section{INTRODUCTION}

Due to its use in radio frequency identification systems, near-field wireless power transfer (WPT) is a research topic that has gained more and more interest over the past years. The research performed on WPT can be divided into three different groups, based on the distance between the antennas employed in the system. First, in the reactive near-field, an often used method to determine the power transfer efficiency (PTE) of a WPT system is by making use of coupled mode theory. In the region of coupled mode resonance, the coupling between the antennas is strong, and a very high PTE can be obtained ( $>80 \%)$ $[1,2]$. However, the coupling distance needs to be very small, and furthermore, an optimal design in this region is very hard because the optimal resonant frequency and the load impedance are strongly dependent on the distance between the antennas. Coupled mode resonance has been widely investigated over the years [3], as well as lateral and angular coil misalignment [4] and multiple-receiver scenarios $[5,6]$.

Second, in the radiative near-field (Fresnel region), beyond the coupled mode resonance region, the PTE decreases rapidly with distance. Nonetheless, for low-power applications such as sensor networks, a sufficiently high PTE can still be obtained. The coupling in this region is much weaker and can be modeled using a spherical mode expansion. This expansion allows the assessment of angular misalignment between the different devices of the WPT system [7]. It is also shown that in order to achieve a PTE close to the theoretical upper bound, electrically small antennas with high radiation efficiency are needed $[7,8]$. The theoretical upper bound has been extended to multiple transmitters in [1].

Third, in the far-field (Fraunhofer region), the PTE can be modeled using the traditional Friis formula [9]. To alleviate orientation dependence of these systems, antennas are designed to be circularly

\footnotetext{
*Correspondence to: Gert-Jan Stockman, IBCN/Electromagnetics Group, Department of Information Technology, Ghent University/iMinds, Sint-Pietersnieuwstraat 41, Ghent, 9000, Belgium.

†E-mail: gertjan.stockman@intec.ugent.be
} 
polarized in order to maximize the polarization efficiency component of the link budget. The question remains if this approach is still suitable when the antennas are brought into the near-field region [3].

To gain better insight in the PTE of WPT systems in the radiative near-field region, several methods developed to mimic the electromagnetic problem with reduced computational complexity are leveraged. Emission modeling is realized using the source reconstruction method or by expanding the electromagnetic field in multipoles [10,11]. Susceptibility modeling focuses more on hybrid techniques such as model reduction combined with full-wave solvers [12]. Finally, domain decomposition methods reduce the computational resources by dividing the electromagnetic problem into different domains. Each of these domains is solved separately using an efficient technique specific to the domain after which the different solutions are combined $[13,14]$. Another method to model the interaction between radiating devices with a low computational complexity is presented in this paper. It is shown that the electromagnetic interaction for arbitrarily positioned devices can be computed using a single simulation (or measurement) of the individual radiation patterns of the devices. This information is sufficient to model any configuration without requiring new simulations every time a device is repositioned. In contrast to [7], these devices are not necessarily electrically small. By taking into account higher-order multipole interactions, any type of antenna may be used (via its radiation pattern). As such, for all possible configurations, our method allows the efficient modeling of the PTE in a realistic WPT system in the radiative near-field (and beyond) with high accuracy.

The structure of this paper is as follows. In Section 2, the formalism of our method is explained. The electromagnetic interaction between a transmitting and receiving device in a WPT setup is described in order to obtain an expression for the wireless link efficiency. Thereto, we also briefly discuss what makes up the rectenna efficiency. Section 3 applies and validates this theory by means of a practical WPT example in the radiative near-field, illustrating how to combine all the different power efficiencies to come to a PTE of the complete WPT system. Moreover, the efficiency of the advocated method is demonstrated. Finally, Section 4 concludes this paper.

In the sequel, all sources and fields are assumed to be time harmonic with angular frequency $\omega$ and with time dependencies $e^{j \omega t}$ suppressed. Unit vectors are denoted with a 'hat', for example, $\hat{\mathbf{v}}$.

\section{FORMALISM}

To obtain the wireless link efficiency between two devices, arbitrarily positioned in space, we first model the electromagnetic interaction between them. We start from the very general problem geometry shown in Figure 1, consisting of a transmitting and receiving device, represented by current density sources $\mathbf{j}_{\mathrm{TX}}$ and $\mathbf{j}_{\mathrm{RX}}$ contained in volumes $V_{\mathrm{TX}}$ and $V_{\mathrm{RX}}$, respectively. To each device's phase center, $\mathcal{O}_{\mathrm{TX}}$ and $\mathcal{O}_{\mathrm{RX}}$, a local coordinate system is attached $\left(\left(x_{\mathrm{TX}}, y_{\mathrm{TX}}, z_{\mathrm{TX}}\right)\right.$ and $\left.\left(x_{\mathrm{RX}}, y_{\mathrm{RX}}, z_{\mathrm{RX}}\right)\right)$. The electric field $\mathbf{e}(\mathbf{r})$ generated by the sources is given by the electric field integral equation (EFIE) [15]:

$$
\mathbf{e}(\mathbf{r})=-j \omega \mu \int_{V} \mathcal{G}\left(\mathbf{r}, \mathbf{r}^{\prime}\right) \cdot \mathbf{j}\left(\mathbf{r}^{\prime}\right) \mathrm{d} \mathbf{r}^{\prime},
$$

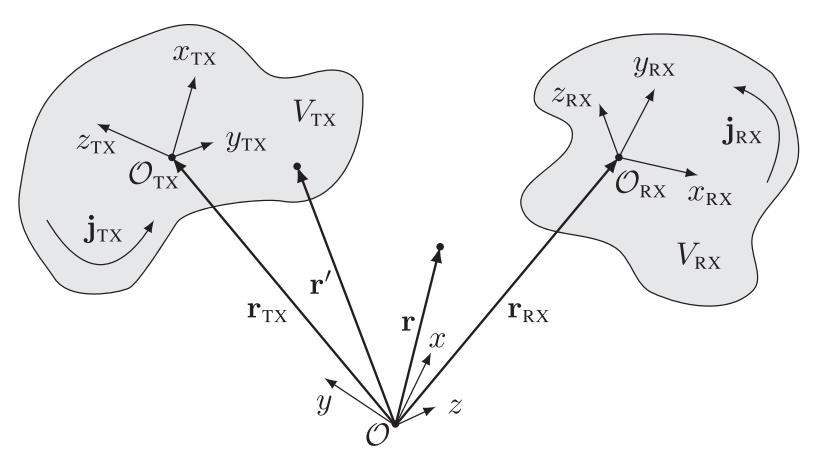

Figure 1. Two devices with arbitrary phase centers $\mathcal{O}_{\mathrm{TX}}$ and $\mathcal{O}_{\mathrm{RX}}$. 
where $\mathbf{j}\left(\mathbf{r}^{\prime}\right)$ represents both current densities $\mathbf{j}_{\mathrm{TX}}\left(\mathbf{r}^{\prime}\right)$ and $\mathbf{j}_{\mathrm{RX}}\left(\mathbf{r}^{\prime}\right)$. $\mu$ is the permeability of the background medium, and $\mathcal{G}\left(\mathbf{r}, \mathbf{r}^{\prime}\right)$ is the three-dimensional dyadic Green's function given by

$$
\mathcal{G}\left(\mathbf{r}, \mathbf{r}^{\prime}\right)=\left[\mathcal{I}+\frac{1}{k^{2}} \nabla \nabla\right] \frac{e^{-j k\left|\mathbf{r}-\mathbf{r}^{\prime}\right|}}{4 \pi\left|\mathbf{r}-\mathbf{r}^{\prime}\right|}
$$

Here, $k=\omega \sqrt{\epsilon \mu}$ is the wavenumber of the background medium and $\epsilon$ its permittivity. The integration domain $V$ extends over both devices (denoted as transmitter TX and receiver RX), that is, $V=V_{\mathrm{TX}} U$ $V_{\mathrm{RX}}$, allowing the decomposition of the EFIE into two components:

$$
\mathbf{e}(\mathbf{r})=-j \omega \mu \int_{V_{\mathrm{TX}}} \mathcal{G}\left(\mathbf{r}, \mathbf{r}^{\prime}\right) \cdot \mathbf{j}_{\mathrm{TX}}\left(\mathbf{r}^{\prime}\right) \mathrm{d} \mathbf{r}^{\prime}-j \omega \mu \int_{V_{\mathrm{RX}}} \mathcal{G}\left(\mathbf{r}, \mathbf{r}^{\prime}\right) \cdot \mathbf{j}_{\mathrm{RX}}\left(\mathbf{r}^{\prime}\right) \mathrm{d} \mathbf{r}^{\prime} .
$$

For devices positioned in each other's Fresnel or Fraunhofer region, the field incident on the receiving device can be accurately approximated by the first term in (3), taking $\mathbf{j}_{\mathrm{TX}}\left(\mathbf{r}^{\prime}\right)$ as the stand-alone current density of the single element. We assume that these devices are spaced sufficiently far from each other so there is no coupling via the reactive near-field.

We proceed by considering the scalar three-dimensional Green's function and by using Gegenbauer's addition theorem $[16,17]$ :

$$
\frac{e^{-j k|\mathbf{D}+\mathbf{d}|}}{|\mathbf{D}+\mathbf{d}|}=-j k \sum_{l=0}^{\infty}(-1)^{l}(2 l+1) j_{l}(k|\mathbf{d}|) h_{l}^{(2)}(k|\mathbf{D}|) P_{l}(\hat{\mathbf{d}} \cdot \hat{\mathbf{D}}) .
$$

Here, $j_{l}(\cdot)$ is the $l$-th order spherical Bessel function of the first kind, $h_{l}^{(2)}(\cdot)$ the $l$-th order spherical Hankel function of the second kind, and $P_{l}(\cdot)$ the Legendre polynomial of degree $l$. The product $j_{l}(k|\mathbf{d}|) P_{l}(\hat{\mathbf{d}} \cdot \hat{\mathbf{D}})$ can be expanded into plane waves as [18]

$$
4 \pi\left(-j^{l}\right) j_{l}(k|\mathbf{d}|) P_{l}(\hat{\mathbf{d}} \cdot \hat{\mathbf{D}})=\iint_{\Omega} e^{-j \mathbf{k} \cdot \mathbf{d}} P_{l}(\hat{\mathbf{k}} \cdot \hat{\mathbf{D}}) \mathrm{d} \hat{\mathbf{k}},
$$

where we integrate over the Ewald sphere $\Omega$ and $\mathbf{k}$ is the wave vector in spherical coordinates:

$$
\mathbf{k}=k \hat{\mathbf{k}}=k(\sin \theta \cos \phi \hat{\mathbf{x}}+\sin \theta \sin \phi \hat{\mathbf{y}}+\cos \theta \hat{\mathbf{z}}) .
$$

In the configuration under study (Figure 1), $\mathbf{r}_{\mathrm{TX}}$ and $\mathbf{r}_{\mathrm{RX}}$ refer to the positions of the phase centers $\mathcal{O}_{\mathrm{TX}}$ and $\mathcal{O}_{\mathrm{RX}}$ of the transmitter and receiver, respectively, with respect to the common origin $\mathcal{O}$. Introducing $\mathbf{D}=\mathbf{r}_{\mathrm{TX}, \mathrm{RX}}=\mathbf{r}_{\mathrm{RX}}-\mathbf{r}_{\mathrm{TX}}, \mathbf{d}=\left(\mathbf{r}-\mathbf{r}_{\mathrm{RX}}\right)-\left(\mathbf{r}^{\prime}-\mathbf{r}_{\mathrm{TX}}\right)$ and thus $(\mathbf{D}+\mathbf{d})=\left(\mathbf{r}-\mathbf{r}^{\prime}\right)$ into (4) and (5) yields an expression for the kernel of the EFIE (1):

$$
\frac{e^{-j k\left|\mathbf{r}-\mathbf{r}^{\prime}\right|}}{\left|\mathbf{r}-\mathbf{r}^{\prime}\right|}=\frac{-j k}{4 \pi} \iint_{\Omega} e^{-j \mathbf{k} \cdot\left(\mathbf{r}-\mathbf{r}_{\mathrm{RX}}\right)} T\left(\mathbf{r}_{\mathrm{TX}, \mathrm{RX}}, \hat{\mathbf{k}}\right) e^{+j \mathbf{k} \cdot\left(\mathbf{r}^{\prime}-\mathbf{r}_{\mathrm{TX}}\right)} \mathrm{d} \hat{\mathbf{k}} .
$$

The operator $T\left(\mathbf{r}_{\mathrm{TX}, \mathrm{RX}}, \hat{\mathbf{k}}\right)$ is calculated as

$$
T\left(\mathbf{r}_{\mathrm{TX}, \mathrm{RX}}, \hat{\mathbf{k}}\right) \approx \sum_{l=0}^{L}(2 l+1) j^{-l} h_{l}^{(2)}\left(k\left|\mathbf{r}_{\mathrm{TX}, \mathrm{RX}}\right|\right) P_{l}\left(\hat{\mathbf{k}} \cdot \hat{\mathbf{r}}_{\mathrm{TX}, \mathrm{RX}}\right) .
$$


The interchange of summation and integration, which was performed to obtain (7), is only allowed if the infinite sum is truncated to a finite number of terms $L$. Traditional guidelines to select this number, which determines the accuracy, are found in [16]. Note that, for (7) to be valid, $|\mathbf{d}|<|\mathbf{D}|$ or equivalently $\left|\left(\mathbf{r}-\mathbf{r}_{\mathrm{RX}}\right)-\left(\mathbf{r}^{\prime}-\mathbf{r}_{\mathrm{TX}}\right)\right|<\left|\mathbf{r}_{\mathrm{TX}, \mathrm{RX}}\right|$ must hold. By taking into account these higher-order multipole interactions, the traditional far-field approximation can be extended into the radiative nearfield with high accuracy. Using the EFIE (1) and the kernel (7), we obtain an approximate expression for the electric field incident on the receiver, caused by the transmitter:

$$
\mathbf{e}^{\mathrm{inc}}(\mathbf{r}) \approx-\frac{\omega k}{4 \pi} \iint_{\Omega} e^{-j \mathbf{k} \cdot\left(\mathbf{r}-\mathbf{r}_{\mathrm{RX}}\right)} T\left(\mathbf{r}_{\mathrm{TX}, \mathrm{RX}}, \hat{\mathbf{k}}\right)[\mathcal{I}-\hat{\mathbf{k}} \hat{\mathbf{k}}] \cdot \mathbf{N}_{\mathrm{TX}}(\hat{\mathbf{k}}) \mathrm{d} \hat{\mathbf{k}},
$$

where $\mathbf{N}_{\mathrm{TX}}(\hat{\mathbf{k}})$ is given by

$$
\mathbf{N}_{\mathrm{TX}}(\hat{\mathbf{k}})=\frac{\mu}{4 \pi} \int_{V_{\mathrm{TX}}} e^{j \mathbf{k} \cdot\left(\mathbf{r}^{\prime}-\mathbf{r}_{\mathrm{TX}}\right)} \mathbf{j}\left(\mathbf{r}^{\prime}\right) \mathrm{d} \mathbf{r}^{\prime}
$$

We define the radiation pattern $\mathbf{F}_{\mathrm{TX}}(\hat{\mathbf{k}})$ of the transmitting device as

$$
\begin{aligned}
\mathbf{F}_{\mathrm{TX}}(\hat{\mathbf{k}}) & =F_{\mathrm{TX}, \theta}(\hat{\mathbf{k}}) \hat{\boldsymbol{\theta}}+F_{\mathrm{TX}, \phi}(\hat{\mathbf{k}}) \hat{\boldsymbol{\phi}} \\
& =j \omega \hat{\mathbf{k}} \times\left[\hat{\mathbf{k}} \times \mathbf{N}_{\mathrm{TX}}(\hat{\mathbf{k}})\right],
\end{aligned}
$$

and hence,

$$
\begin{aligned}
{[\mathcal{I}-\hat{\mathbf{k}} \hat{\mathbf{k}}] \cdot \mathbf{N}_{\mathrm{TX}}(\hat{\mathbf{k}}) } & =\mathbf{N}_{\mathrm{TX}}(\hat{\mathbf{k}})-\hat{\mathbf{k}}\left[\hat{\mathbf{k}} \cdot \mathbf{N}_{\mathrm{TX}}(\hat{\mathbf{k}})\right] \\
& =-\hat{\mathbf{k}} \times\left[\hat{\mathbf{k}} \times \mathbf{N}_{\mathrm{TX}}(\hat{\mathbf{k}})\right] \\
& =-\frac{1}{j \omega} \mathbf{F}_{\mathrm{TX}}(\hat{\mathbf{k}}) .
\end{aligned}
$$

Consequently, the incident electric field (9) can be written in terms of $\mathbf{F}_{\mathrm{TX}}(\hat{\mathbf{k}})$.

Assume now, for simplicity, that the transmitter and receiver in our WPT configuration are oneport devices such as single antenna elements. Then, an equivalent circuit representation [15] of a WPT system is shown in Figure 2. Here, $Z_{\mathrm{TX}}$ and $Z_{\mathrm{RX}}$ are the radiation impedances of the transmit and receive antennas, respectively. The transmitter is driven by means of a Thévenin generator composed of a sinusoidal voltage source $V_{g}$ with internal impedance $Z_{g}$. The Norton equivalent of the receiver consists of a load impedance $Z_{L}$ and a short-circuit current $I_{s c}$, computed as
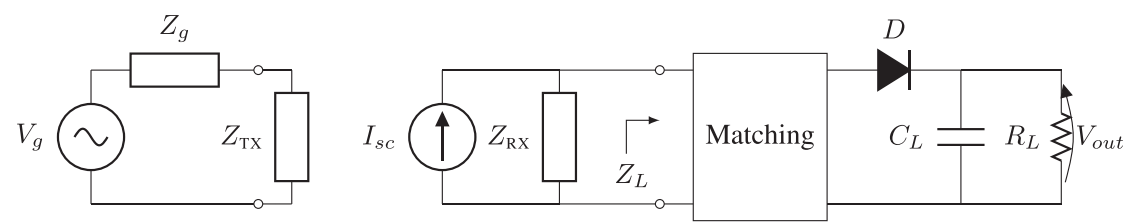

Figure 2. Equivalent circuit of a wireless power transfer (WPT) link, that is, a transmit antenna and a receive antenna with rectifier (rectenna). 


$$
I_{s c}=-\frac{1}{V_{0}} \int_{V_{\mathrm{RX}}} \mathbf{e}^{\mathrm{inc}}(\mathbf{r}) \cdot \mathbf{j}(\mathbf{r}) \mathrm{d} \mathbf{r},
$$

where $V_{0}$ is the pertinent normalization factor, evolving from the reciprocity theorem, depending on the normalized radiation pattern when operating the antenna in transmit mode (see [19] for a detailed explanation). As the antennas' radiation patterns scale linearly with $V_{0}$, this parameter can be chosen to be $1 \mathrm{~V}$. Defining the radiation pattern $\mathbf{F}_{\mathrm{RX}}(\hat{\mathbf{k}})$ of the receiver in a similar way as (11) and substituting (9) and (12) into (13) yield the following expression for the short-circuit current:

$$
I_{s c} \approx-\frac{1}{Z V_{0}} \iint_{\Omega} T\left(\mathbf{r}_{\mathrm{TX}, \mathrm{RX}}, \hat{\mathbf{k}}\right) \mathbf{F}_{\mathrm{TX}}(\hat{\mathbf{k}}) \cdot \mathbf{F}_{\mathrm{RX}}(-\hat{\mathbf{k}}) \mathrm{d} \hat{\mathbf{k}},
$$

where $Z=\sqrt{\frac{\mu}{\epsilon}}$ is the wave impedance of the background medium.

The aforementioned result allows us to calculate the short-circuit current of a receiving antenna by using only the single element radiation patterns $\mathbf{F}_{\mathrm{TX}}(\hat{\mathbf{k}})$ and $\mathbf{F}_{\mathrm{RX}}(\hat{\mathbf{k}})$ of transmit and receive antennas, respectively. The operator $T\left(\mathbf{r}_{\mathrm{TX}, \mathrm{RX}}, \hat{\mathbf{k}}\right)$ allows efficient translations between the two antennas. When devices are repositioned in space, only the original radiation patterns are needed. The interaction between devices is efficiently calculated for any set of positions of the devices, whereas for traditional simulation tools or measurements, a new computation or measurement is needed for every repositioning [20]. Our method also allows the use of electrically large antennas by taking into account higher-order multipole interactions.

Using the expression for the short-circuit current (14), the power delivered to the receiving antenna's load $Z_{L}$ is written as

$$
P_{\mathrm{RX}}=\frac{1}{2} \Re\left(Z_{L}\left|I_{s c}\right|^{2}\left|\frac{Z_{\mathrm{RX}}}{Z_{\mathrm{RX}}+Z_{L}}\right|^{2}\right) .
$$

Knowing that the power emitted by the transmitter with radiation pattern $\mathbf{F}_{\mathrm{TX}}(\hat{\mathbf{k}})$ is calculated as

$$
P_{\mathrm{TX}}=\frac{1}{2 Z} \iint_{\Omega}\left|\mathbf{F}_{\mathrm{TX}}(\hat{\mathbf{k}})\right|^{2} \mathrm{~d} \hat{\mathbf{k}}
$$

the power efficiency $\eta_{\text {link }}$ of the wireless link between transmitter and receiver is described by

$$
\eta_{\text {link }}=\frac{P_{\mathrm{RX}}}{P_{\mathrm{TX}}}=\frac{\frac{1}{2} \Re\left(Z_{L}\left|I_{s c}\right|^{2}\left|\frac{Z_{\mathrm{RX}}}{Z_{\mathrm{RX}}+Z_{L}}\right|^{2}\right)}{\frac{1}{2 Z} \iint_{\Omega}\left|\mathbf{F}_{\mathrm{TX}}(\hat{\mathbf{k}})\right|^{2} \mathrm{~d} \hat{\mathbf{k}}} .
$$

In the context of WPT, an interesting conclusion is now readily derived from (14) and (17) to calculate the wireless link efficiency $\eta_{\text {link }}$ between a transmitting device and a receiving device; it is sufficient to have access to either their measured or simulated radiation patterns. This is true for any relative position between the two devices, indicated by $\mathbf{r}_{\mathrm{TX}, \mathrm{RX}}$, as long as there is no coupling via the reactive near-field, hence $\left|\mathbf{r}_{\mathrm{TX}, \mathrm{RX}}\right|$ should be larger than, say, at least a sixth of the wavelength.

The PTE of a WPT system such as the one schematically shown in Figure 2 is defined as the ratio of direct current (DC) power dissipated in the load $R_{L}$ at the receiving side to the alternating current (AC) power delivered to the transmit antenna. Assuming no radiation losses, this AC power equals 
$P_{\mathrm{TX}}$ given by (16). The DC power delivered to the load is found by combining the link efficiency calculated in (17) with the efficiency of the rectenna. Here, it is important to note that the latter is not only made up by the efficiency of the rectifier but also by the efficiency of a potential matching circuit between the antenna and the rectifier. Because the input impedance $Z_{L}$ of nonlinear circuits depends on the incoming power, this matching efficiency $\eta_{\text {match }}$ will also be a function of the incoming power. The matching efficiency can, for a known rectifier circuit, be simulated using commercial tools such as advanced design system (ADS). The efficiency of the rectifier circuit itself can be calculated by comparing the DC power, delivered to the load resistor $R_{L}$, to the incoming power $P_{\text {inc }}=\eta_{\text {match }} \cdot P_{\mathrm{RX}}$, injected into the rectifier circuit. The DC power $P_{D C}$ delivered to the load $R_{L}$ is calculated as

$$
P_{D C}=\frac{V_{o u t}^{2}}{R_{L}}
$$

The DC output voltage $V_{\text {out }}$ may be found from simulation tools such as ADS or from an analytical expression describing the behavior of diode detectors [21]. By clever alteration of the parameters of such expressions, also voltage doublers can be taken into account [22].

\section{APPLICATION TO A WIRELESS POWER TRANSFER SYSTEM}

\subsection{Description of the wireless power transfer system}

To demonstrate the efficiency and appositeness of the formalism, an application example is considered consisting of a multi-receiver scenario. In this example, a large standard gain horn (SGH) antenna is applied as transmitter, transferring power to an array of patch antennas. These receiving antennas are embedded into a wall in order to supply power to a sensor network (Figure 3). Each patch antenna is a dual polarized textile antenna for operation in the industrial, scientific, and medical (ISM) band at frequency $f=2.45 \mathrm{GHz}$ [23]. The antennas in the array are spaced sufficiently apart such that their mutual coupling is negligible (the separation distance between phase centers is minimally one wavelength $\lambda$ ). The patch antennas are located inside a brick wall as shown in Figure 4 . There is $1 \mathrm{~cm}$ of

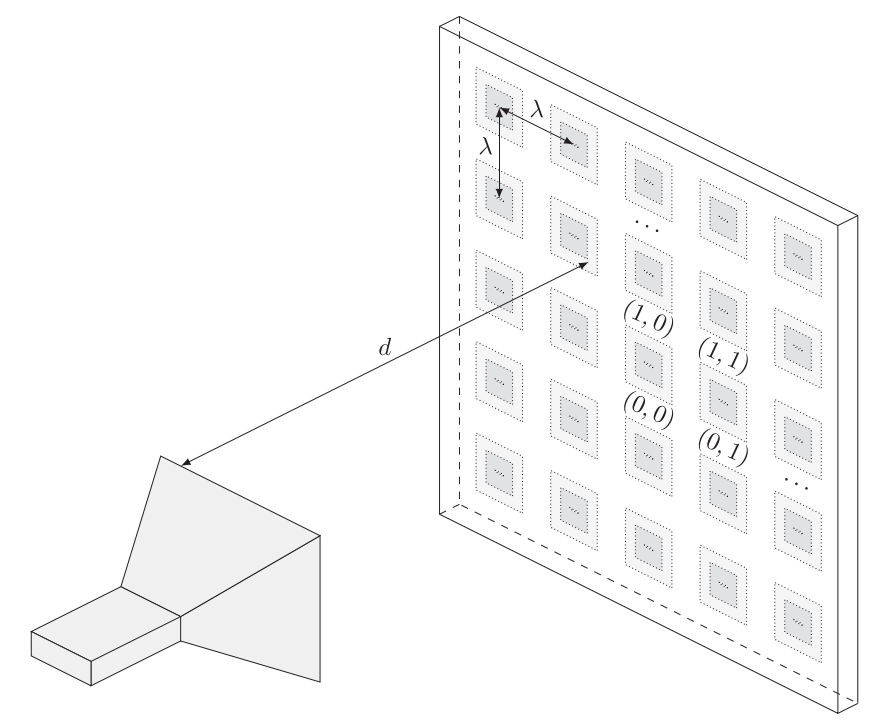

Figure 3. An antenna array embedded into a brick wall used to wirelessly power a sensor network. The antenna elements are dual-polarized textile patch antennas, spaced at least one wavelength $\lambda$ from each other. The transmitting antenna is a standard gain horn (SGH) transmitting at $f=2.45 \mathrm{GHz}$. It is separated from the brick wall by a distance $d$. 


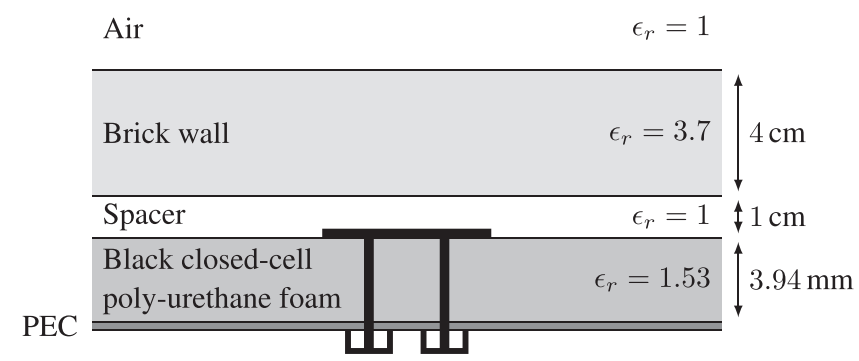

Figure 4. Cross section of the dual polarized textile antenna embedded inside a brick wall with a perfect electrically conducting ground plane (not on scale). More information on the antenna is found in [23].

spacer in front of the antenna followed by $4 \mathrm{~cm}$ of brick wall $\left(\epsilon_{r}=3.7\right.$ and $\mu_{r}=1$ [24]). The radiation pattern of a single antenna element embedded into the brick wall is calculated using ADS-momentum. Using an analytical expression for the radiation pattern of the horn antenna (see the appendix of [20], where here, we chose $a=369 \mathrm{~mm}, b=273 \mathrm{~mm}, \rho_{1}=344 \mathrm{~mm}$, and $\rho_{2}=363 \mathrm{~mm}$ for our SGH1.7). The SGH's phase center is aligned to the phase center of the center antenna element of the array (= antenna element $(0,0))$. The negligible coupling between antenna elements is confirmed by a fullwave simulation, as the mutual coupling between any two elements in the array is always less than $-25 \mathrm{~dB}$ (with the brick wall and possible surface wave coupling taken into account).

\subsection{Validation example}

In order to validate the proposed formalism, we first consider an example where the horn antenna is used as transmitter and a dual polarized patch antenna as receiver. The results of measurements and simulations of the transmission coefficient $S_{21}$ will be compared. The transmission coefficient is relevant in this discussion because it is directly related to the efficiency of the wireless link as $\eta_{\text {link }}=\left|S_{21}\right|^{2}$. The brick wall is omitted for ease of measurement, but this does not detract from the generality of the method. The measurement has been performed in an anechoic chamber and a vector network analyzer, that is, the PNA-X N5242A of Agilent Technologies (Santa Clara, CA, USA) is hereby used. The distance between the transmit and receive antennas $d$ is varied up to $2 \lambda$, to cover the radiative near-field. The obtained results are shown in Figure 5, where also a comparison with the Friis formula is presented. Simulations (with $L=15$ ) using the advocated formalism and measurements yield an absolute error of only $1 \mathrm{~dB}$, which can be attributed to the lossless horn antenna used in the simulations and to horn, connector, and cable loss in the measurements. It is clear that, in the reactive near-field, the traditional Friis formula produces a huge overestimation and that it is only usable in the far-field, where it indeed converges to our novel formalism.

\subsection{Calculation of the power transfer efficiency}

To calculate the PTE for the multi-receiver scenario in Figure 3, we first compute the power efficiency of the link $\eta_{\text {link }}$ using the formalism explained in Section 2. The novel method easily allows a repositioning of the used antennas which, for example, allows a sweep of the wireless link efficiency over different separation distances $d$ between horn and the antennas in the wall. The resulting efficiency in function of the separation distance is shown in Figure 6. Element $(0,0)$ is the center element that is perfectly aligned with the phase center of the horn antenna. Element $(0,1)$ is located one wavelength to the right of the center element, and elements $(2,0)$ and $(2,1)$ are located two wavelengths above elements $(0,0)$ and $(0,1)$, with one row of antennas in between. For certain antenna elements that are not aligned to the phase center of the SGH, an optimal distance $d_{o p t}$ exists where the wireless link efficiency is at its highest. For the aligned element (the center element), the efficiency of the link increases monotonically with decreasing separation distance $d$. For large distances $d$, all efficiencies converge to the same value, which is also the value predicted by the traditional Friis formula. The advantage 


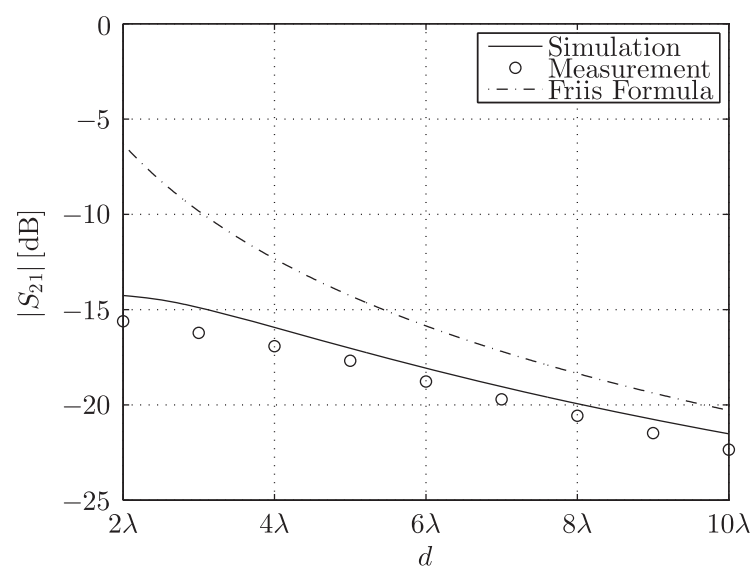

Figure 5. Comparison of simulation, measurement, and Friis formula of $S_{21}$ in a single-receiver scenario.

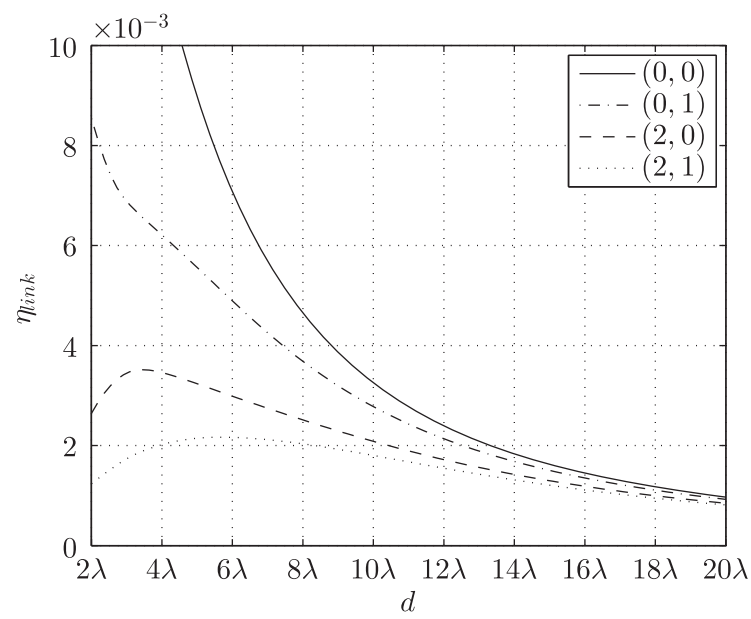

Figure 6. Efficiency of the wireless link $\eta_{\text {link }}$ between the transmitting standard gain horn (SGH) and several receiving antenna elements embedded into the wall.

of our method is that by accounting for higher-order multipole interactions, a high accuracy is also obtained in the radiative near-field (Fresnel region) whereas the Friis formula can only lead to good results when the two devices are situated in each others far-field (Fraunhofer zone).

In this example, simulations have been carried out on an Intel ${ }^{\circledR}$ core $^{\mathrm{TM}} \mathrm{i7}-2600$ processor (Intel Corporation, Santa Clara, CA, USA) running at $3.40 \mathrm{GHz}$ with $16 \mathrm{~GB}$ of memory. Calculation of the link efficiencies of the $5 \times 5$ elements of the antenna array using (17) for one fixed separation distance $d$ between SGH and the wall only takes $0.25 \mathrm{~s}$. This indicates the computational efficiency of our method. Indeed, for different antenna elements of the array, only the translation operator $T\left(\mathbf{r}_{\mathrm{TX}, \mathrm{RX}}, \hat{\mathbf{k}}\right)$ has to be recalculated.

In a complete WPT system, every antenna element embedded in the wall is used as rectenna by connecting it via a matching circuit to a voltage doubler and rectifier circuit. This circuit converts the incoming AC power to DC power that is delivered to the load $R_{L}$. To obtain the complete PTE of the WPT system, the wireless link efficiency $\eta_{\text {link }}$ should now be combined with the rectenna efficiency [22]. To compute the latter, a rectenna is designed and simulated in ADS. The circuit is shown in Figure 7, and it is an often used configuration for rectennas used in WPT systems. The receiving antenna element is replaced by its Norton equivalent. The resulting short-circuit current $I_{s c}$ is given by (14), and the input impedance $Z_{\mathrm{RX}}$ is found using ADS. The antenna and the voltage doubler and rectifier circuit are matched using an inductor $L_{m}=5 \mathrm{nH}$. The voltage doubler and rectifier circuit 


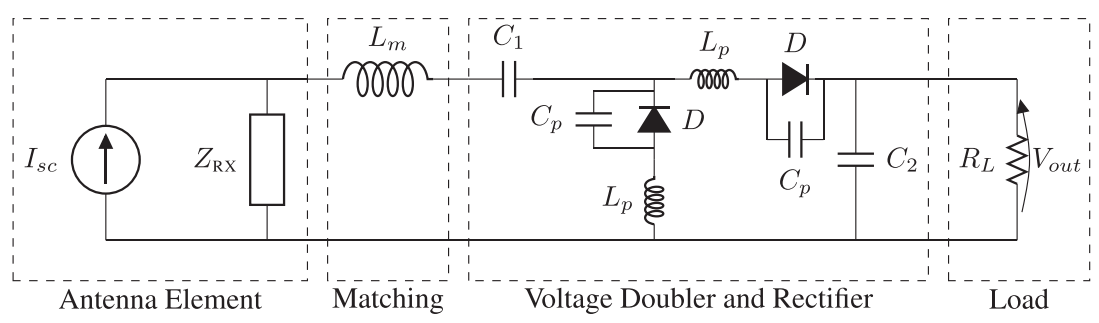

Figure 7. The complete schematic of a rectenna element as designed and simulated in advanced design system (ADS).

itself consists of two HSMS-2850 Schottky diodes together with their package parasitics $C_{p}=0.08 \mathrm{pF}$ and $L_{p}=2 \mathrm{nH}$. For these diodes, a pertinent Spice model was used. The capacitors used are $C_{1}=$ $C_{2}=100 \mathrm{pF}$, and this example was designed for a load resistance of $R_{L}=100 \Omega$. The matching circuit was designed to have optimal matching for $P_{\mathrm{RX}}=-10 \mathrm{dBm}$. Because the input impedance of the voltage doubler and rectifier circuit depends on incoming power, due to the nonlinear diodes, the matching efficiency $\eta_{\text {match }}$ also depends on the incoming power $P_{\mathrm{RX}}$. Using a harmonic balance simulation in ADS, the input impedance of the voltage doubler and rectifier circuit is calculated for different incoming powers, from which the matching efficiency is then calculated as

$$
\begin{aligned}
\eta_{\text {match }} & =1-|\Gamma|^{2} \\
& =1-\left|\frac{Z_{L}-Z_{\mathrm{RX}}^{*}}{Z_{L}+Z_{\mathrm{RX}}}\right|^{2} .
\end{aligned}
$$

Here, $Z_{L}$ is the input impedance of the matching circuit, which depends on $P_{\mathrm{RX}}$. The resulting matching efficiency is shown in Figure 8. Furthermore, the efficiency of the voltage doubler and rectifier circuit $\eta_{\text {rect }}$ is simply calculated by dividing the DC power delivered to the load by the AC power delivered to the input of the circuit. The total efficiency $\eta_{t o t}$ of the rectenna is found by combining both efficiencies as $\eta_{\text {tot }}=\eta_{\text {match }} \cdot \eta_{\text {rect }}$. The results shown in Figure 8 are similar to results shown in Figures 7 and 10 in [22], indicating that we also consider realistic voltage doubler and rectifier circuit.

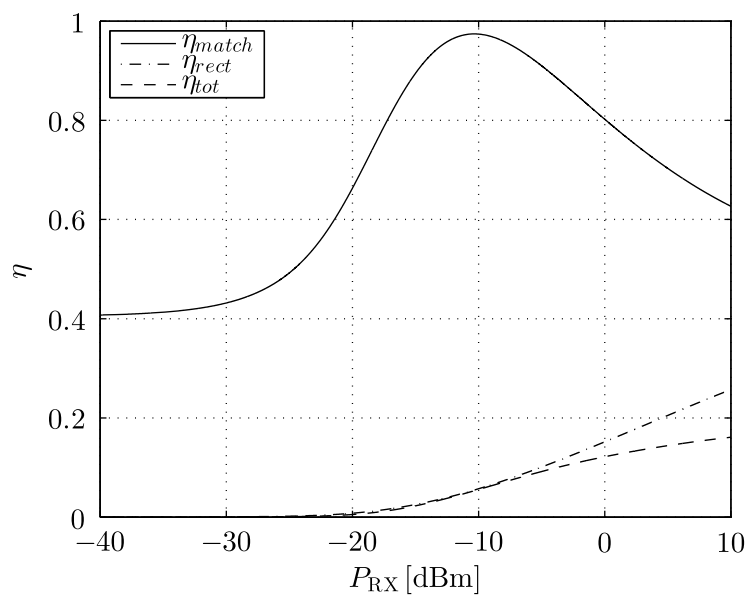

Figure 8. Matching efficiency $\left(\eta_{\text {match }}\right)$, voltage doubler and rectifier efficiency $\left(\eta_{\text {rect }}\right)$, and total efficiency $\left(\eta_{\text {tot }}\right)$ of the rectenna. The matching network was designed for optimal matching when $P_{\mathrm{RX}}=-10 \mathrm{dBm}$. The matching efficiency and the voltage doubler and rectifier efficiency are calculated using a harmonic balance simulation in advanced design system (ADS). The total efficiency of the rectenna is calculated as $\eta_{\text {tot }}=$

$$
\eta_{\text {match }} \cdot \eta_{\text {rect }} \text {. }
$$




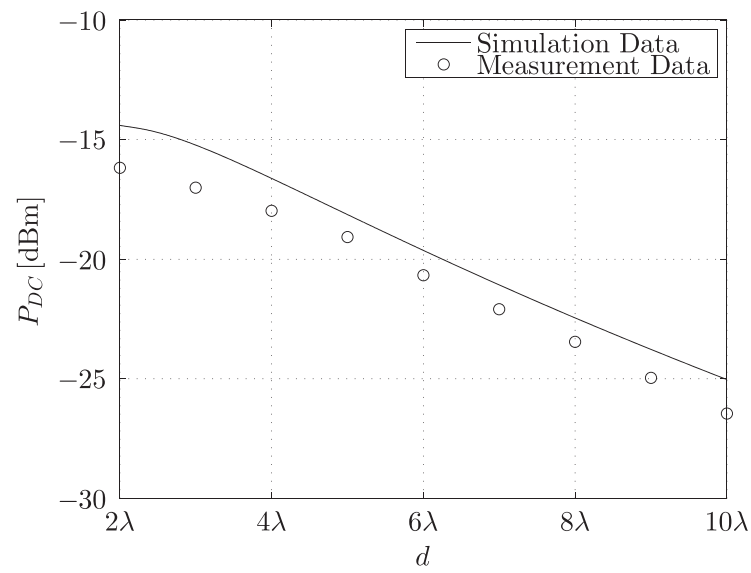

Figure 9. Direct current (DC) power delivered to the load of the center antenna element for measured and simulated data of the wireless link efficiency when the power delivered to the standard gain horn (SGH) is $P_{S G H}=10 \mathrm{dBm}$ and no brick wall is present.

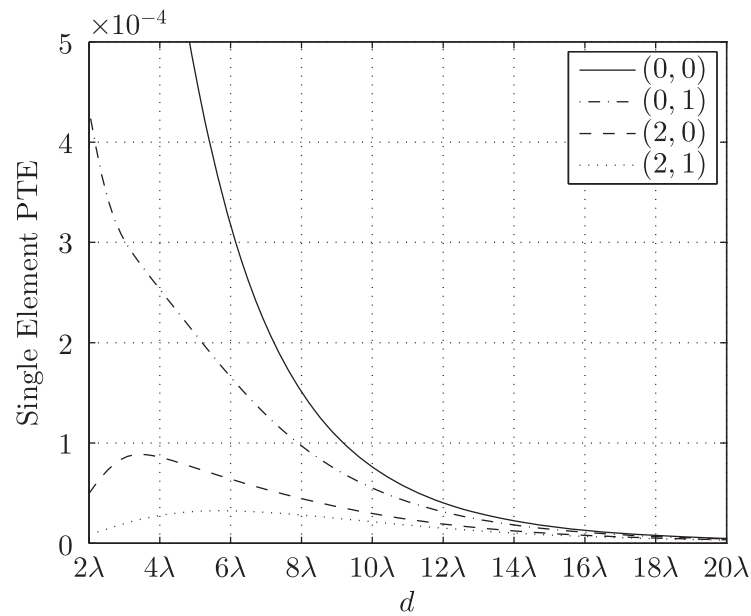

Figure 10. The power transfer efficiency (PTE) between the transmitting standard gain horn (SGH) and different receiving antenna elements embedded into the wall. For elements not aligned with the phase center of the SGH, an optimal distance is again observed.

Using this voltage doubler and rectifier circuit on the measured and simulated data for the example considered in Figure 5 ( $P_{S G H}=10 \mathrm{dBm}$, no brick wall), the DC power delivered to the load of the center antenna element is found and shown in Figure 9. This again validates the method as a good agreement is observed. The error of about $1 \mathrm{~dB}$ is again attributed to losses of the horn antenna, connectors, and cables, which are not taken into account in the simulation. The total PTE of a single antenna element in the array inside the brick wall is found by combining the wireless link efficiency and the rectenna efficiency. The result is shown in Figure 10 for different antenna elements. The power delivered to the transmitting SGH is again $P_{S G H}=10 \mathrm{dBm}$. For the center element, the efficiency rises with a lower separation distance $d$, but for certain other (misaligned) elements, there is an optimal distance $d_{\text {opt }}$ at which the PTE becomes maximal. The large difference in PTE between the different elements of the array is obviously due to the directive nature of the radiation pattern of the SGH. If a more omnidirectional transmit antenna was chosen, the efficiency of the different elements would be more similar. It is now possible to obtain an efficiency of the complete WPT system by combining all the different single element PTEs. The result is shown in Figure 11 where the PTE of the complete system is given by 


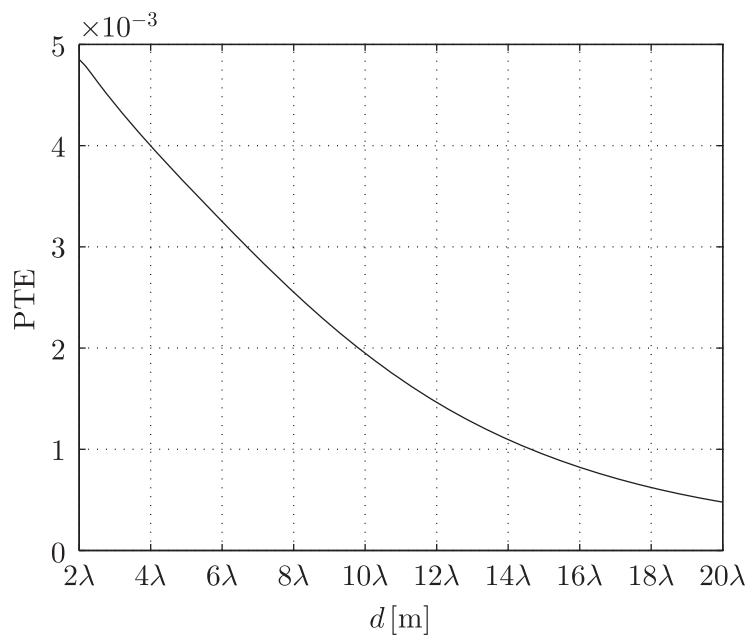

Figure 11. The total power transfer efficiency (PTE) of the complete wireless power transfer (WPT) system. This includes the link efficiency, matching efficiency, and efficiency of the voltage doubler and rectifier circuit.

$$
\mathrm{PTE}=\frac{\sum_{i, j} P_{D C, i j}}{P_{\mathrm{TX}}}
$$

with $P_{D C, i j}$ the DC power delivered to the load of rectenna element $(i, j=-2,-1,0,1,2)$.

\section{CONCLUSION}

In this paper, we have presented a method to calculate the PTE of a WPT system in the radiative near-field. The method is computationally effective as it merely relies only on a single simulation (or measurement) of the radiation pattern of the antennas in the system. As long as there is no coupling via the reactive near-field between two transmitters, repositioning of the antennas can be carried out without large additional cost, making our method suitable for multi-receiver scenarios, as was abundantly shown in the application example. This example also demonstrated that investigating the behavior of WPT systems in the radiative near-field is imperative as the PTE does not always monotonically decrease with increasing distance between the antennas. Instead, for certain antenna elements, an optimal distance where the PTE is maximal is found, and the designers and users should be aware of this.

\section{REFERENCES}

1. Yoon IJ, Ling H. Investigation of near-field wireless power transfer under multiple transmitters. IEEE Antennas and Wireless Propagation Letters 2011; 10:662-665.

2. Yoon IJ, Ling H. Investigation of near-field wireless power transfer in the presence of lossy dielectric materials. IEEE Transactions on Antennas and Propagation 2013; 61(1):482-488.

3. Yoon IJ, Ling H. Design of an electrically small circularly polarised turnstile antenna and its application to near-field wireless power transfer. IET Microwaves,
Antennas Propagation 2014; 8(5): 308-314.

4. Fotopoulou K, Flynn B. Wireless power transfer in loosely coupled links: coil misalignment model. IEEE Transactions on Magnetics 2011; 47(2): 416-430.

5. Cannon B, Hoburg J, Stancil D, Goldstein S. Magnetic resonant coupling as a potential means for wireless power transfer to multiple small receivers. IEEE Transactions on Power Electronics 2009; 24(7):1819-1825.

6. Kurs A, Moffatt R, Soljacic M. Simultaneous mid-range power transfer to multiple devices. Applied Physics Letters 2010; 96(4):044 102-044 102-3.

7. Lee J, Nam S. Fundamental aspects of near-field coupling small antennas for wireless power transfer. IEEE Transactions on Antennas and Propagation 2010; 58(11):3442-3449.

8. Yoon IJ, Ling H. Realizing efficient wireless power transfer using small folded cylindrical helix dipoles. IEEE Antennas and Wireless Propagation Letters 2010; 9:846-849.

9. Balanis C. Antenna Theory: Analy-sis and Design. Wiley: Hoboken, 1982. 
10. Li P, Jiang LJ. Source reconstruction method-based radiated emission characterization for PCBs. IEEE Transactions on Electromagnetic Compatibility 2013; 55(5):933-940.

11. Kralicek P, John W, De Smedt $\mathrm{R}$, Vervoort K, Garbe H. A voltage controlled emission model of electromagnetic emission of IC for system analysis, IEEE International Symposium on Electromagnetic Compatibility, Montreal, Quebec, 2001; 1197-1202.

12. Erdin I, Nakhla M, Achar R. Circuit analysis of electromagnetic radiation and field coupling effects for networks with embedded fullwave modules. IEEE Transactions on Electromagnetic Compatibility 2000; 42(4):449-460.

13. Shao Y, Peng Z, Lee JF. Signal integrity analysis of high-speed interconnects by using nonconformal domain decomposition method. IEEE Transactions on Components, Packaging and Manufacturing Technology 2012; 2(1):122-130.

14. Peng Z, Lim KH, Lee JF. Nonconformal domain decomposition methods for solving large multiscale electromagnetic scattering problems. Proceedings of the IEEE 2013; 101(2):298-319.

15. Van Bladel J. Electromagnetic Fields. John Wiley \& Sons: Hoboken, 2007.

16. Chew WC, Jin JM, Michielssen $\mathrm{E}$, Song J. Fast and Efficient Algorithms in Computational Electromagnetics. Artech House Publishers: Norwood, 2001.

17. Gibson WC. The Method of Moments in Electromagnetics. Chapman \& Hall/CRC: Boca Raton, London and New York, 2008.

18. Stratton J. Electromagnetic Theory. McGraw-Hill: New York, 1941.

19. Van Bladel J. On the equivalent circuit of a receiving antenna. IEEE Antennas and Propagation Magazine 2002; 44(1):164-165.

20. Stockman GJ, Rogier H, Vande Ginste D. Efficient modeling of interactions between radiating devices with arbitrary relative positions and orientations. IEEE Transactions on Electromagnetic
Compatibility 2014; $\quad \mathbf{5 6}(6)$ : 1313-1321.

21. Harrison R, Le Polozec $X$. Nonsquare law behavior of diode detectors analyzed by the Ritz-Galerkin method. IEEE Transactions on Microwave Theory and Techniques 1994; 42(5): 840-846.

22. Visser H, Vullers R. RF energy harvesting and transport for wireless sensor network applications: principles and requirements. Proceedings of the IEEE 2013; 101(6): 1410-1423.

23. Vallozzi L, Rogier H, Hertleer C. Dual polarized textile patch antenna for integration into protective garments. IEEE Antennas and Wireless Propagation Letters 2008; 7:440-443.

24. Stavrou S, Saunders S. Review of constitutive parameters of building materials, Twelfth International Conference on Antennas and Propagation, 2003. (ICAP 2003), Vol. 1, University of Exeter, United Kingdom, 2003; 211-215 vol.1. 